\title{
Bistatic Synthetic Aperture Radar Imaging for Arbitrary Trajectories in the Presence of Noise and Clutter
}

\author{
John Swoboda $^{a}$, Can Evren Yarman ${ }^{b}$ and Birsen Yazıc1 ${ }^{c}$ \\ ${ }^{a}$ MITRE Corporation, Bedford MA 01730-1420; \\ ${ }^{b}$ Houston Technology Center, WesternGeco-Schlumberger, Houston, TX 77042; \\ ${ }^{c}$ Electrical, Computer and System Engineering Department,Rensselaer Polytechnic Institute, \\ Troy, NY 12180
}

\begin{abstract}
We present an analytic, filtered-backprojection (FBP) type inversion method for bistatic synthetic aperture radar (BISAR) when the measurements have been corrupted by noise and clutter. The inversion method uses microlocal analysis in a statistical setting to design a backprojection filter that reduces the impact of noise and clutter while preserving the fidelity of the target image. We assume an isotropic single scattering model for the electromagnetic radiation that illuminates the scene of interest. We assume a priori statistical information on the target, clutter and noise. We demonstrate the performance of the algorithm and its ability to better resolve targets through numerical simulations.
\end{abstract}

Keywords: Bistatic, filtered-backprojection, microlocal analysis, radar, synthetic aperture imaging, noise, clutter

\section{INTRODUCTION}

Synthetic aperture radar (SAR) uses the measurements from scattered electromagnetic waves to reconstruct an image of a scene of interest. These electromagnetic waves are transmitted from an antenna that is mounted on a satellite or aircraft.

In the specific case of bistatic SAR (BISAR) the transmitting and receiving antennas are located on separate aircraft, unlike monostatic SAR in which the antennas are co-located. ${ }^{1}$ This adds a tactical advantage by separating the bulky and expensive transmitter from the smaller and more expendable receiver while also giving a greater resistance to counter measures developed for monostatic SAR. ${ }^{2}$

There are many well-known algorithms in the literature that can reconstruct a SAR image when a narrow beam approximation can be used. ${ }^{3-8}$ This approximation is not always valid in many systems where the antenna has a large footprint and poor directivity. Examples of this can be found in foliage-penetrating radar ${ }^{9,10}$ where it is often necessary to use low frequency waveforms, which are difficult to focus into a narrow beam.

Recently, microlocal techniques have been used to develop new reconstruction algorithms for SAR. ${ }^{11-14}$ Microlocal analysis is a mathematical study of singularities and associated high frequency behavior. It involves the study of Fourier integral operators (FIOs). ${ }^{15-23}$ It leads to methods and techniques for the approximate inversion of FIOs. When these techniques are applied to SAR reconstruction, the algorithms that are created correctly place visible edges in the scene at the correct location, orientation and strength. Additionally, these inversion techniques can account for nonideal imaging conditions such as non-flat topography and arbitrary flight trajectories.

It has also been shown that statistical information can be incorporated into the inversion algorithms derived from microlocal methods. ${ }^{24,25}$ These reconstruction schemes have the advantage of being non-iterative. There

Further author information: (Send correspondence to B.Y.)

C.E.Y.: E-mail: yarman@ecse.rpi.edu

B.Y.: E-mail:yazici@ecse.rpi.edu

J.S.: E-mail:jswoboda@mitre.org

Airborne Intelligence, Surveillance, Reconnaissance (ISR) Systems and Applications VI, edited by Daniel J. Henry, Proc. of SPIE Vol. 7307, 73070D · C 2009 SPIE · CCC code: 0277-786X/09/\$18 · doi: 10.1117/12.818609 
has been other work in the area of edge preserving algorithms that use statistical information as well. ${ }^{26,27}$ Unlike the microlocal approach, these schemes result in iterative and computationally intensive algorithms.

This work is concerned with the derivation of a method and algorithm for the reconstruction of a BISAR image when the measurements have been corrupted by noise and clutter. We assume the transmitter and receiver are on arbitrary, but known flight paths over a known and flat topography; and that the statistics of the target, clutter and noise are known a priori. We derive a noise and clutter suppression filter using microlocal techniques. This specific filter will reduce the mean squared error (MSE) of the image through finding the minimum variance estimate of the target. The numeric implementation of the algorithm will be discussed and simulation results will be shown to demonstrate its performance.

The organization of the paper is as follows. Section 2 presents the forward model, along with the statistics of scene and noise. Section 3 details the derivation of the algorithm, specifically the image formation and design of the backprojection filter. In Section 4 we present our simulation set up and results. Lastly, conclusions can be found in Section 5 .

\section{FORWARD MODEL FOR BISAR DATA}

We first discuss the development of the forward model for the SAR data in ideal conditions; and next we present the statistical model with the additive noise and clutter.

\subsection{Forward Model}

Let $\psi: \mathbb{R}^{2} \rightarrow \mathbb{R}$ represent the ground topography and $T(\mathbf{x}), \mathbf{x}=\left[x_{1}, x_{2}, \psi\left(x_{1}, x_{2}\right)\right]^{T}$ represent the ground reflectivity function. We assume that the electromagnetic waves scatter on an infinitesimally small region on the surface, thus allowing $T(\mathbf{x})$ to be a function of only two variables, $\boldsymbol{x}=\left[x_{1}, x_{2}\right]^{T}$.

Let $\gamma_{T}(s), \gamma_{R}(s) \in \mathbb{R}^{3}, s \in \mathbb{R}$ represent the transmitter and receiver trajectories, respectively. Under the single scattering (Born) approximation the ideal received data $d(s, t)$, can be modeled as the following: ${ }^{14}$

$$
d(s, t) \approx \mathcal{F}[T](s, t):=\int \mathrm{e}^{-\mathrm{i} 2 \pi v\left(t-R_{T R}(s, \mathbf{x}) / c_{0}\right)} A_{T R}(\boldsymbol{x}, s, v) T(\boldsymbol{x}) d v d \boldsymbol{x},
$$

where $R_{T R}(s, \mathbf{x})=\left|\gamma_{T}(s)-\mathbf{x}\right|+\left|\mathbf{x}-\gamma_{R}(s)\right|$ is the bistatic range, which is the total traveled distance from the transmitter to point $\mathbf{x}$ in the scene and from $\mathbf{x}$ to the receiver at $\gamma_{R}(s), v$ is the temporal frequency, $t$ is the fast-time variable, $s$ is the slow-time variable and $c_{0}$ is the speed of light in free space. The term $A_{T R}$ is a complex amplitude function that represents the transmitted waveform, transmitter and receiver antenna beam patterns, geometrical spreading factors, etc. In the case where an isotropic antenna is transmitting a waveform, $P(v)$, to an isotropic receiver antenna, $A_{T R}(\boldsymbol{x}, s, v)$ becomes $P(v)\left(4 \pi\left|\boldsymbol{\gamma}_{T}(s)-\mathbf{x}\right|\left|\mathbf{x}-\gamma_{R}(s)\right|\right)^{-1} \cdot{ }^{12,14,25}$

We make the assumption that for some $m_{A}, A_{T R}$ satisfies the following symbol estimate ${ }^{11,12}$

$$
\sup _{(s, \boldsymbol{x}) \in K}\left|\partial_{v}^{\alpha} \partial_{s}^{\beta} \partial_{x_{1}}^{\rho_{1}} \partial_{x_{2}}^{\rho_{2}} A_{T R}(\boldsymbol{x}, s, v)\right| \leq C_{0}\left(1+v^{2}\right)^{\left(m_{A}-|\alpha|\right) / 2}
$$

where $K$ is any compact subset of $\mathbb{R} \times \mathbb{R}^{2}$, and the constant $C_{0}$ depends on the integers $\alpha, \beta, \rho_{1}$ and $\rho_{2}$. This assumption is necessary when using the method of stationary phase and makes the forward operator $\mathcal{F}$, an FIO. The assumption is valid when a bandlimited waveform is transmitted and received over broadband antennas. 


\subsection{Statistical model for noise and clutter}

In practical radar systems, the presence of noise and clutter is inevitable in received signals. In this subsection we describe our model in the presence of these unwanted components as well as discuss the statistical properties of the data.

In radar applications the scene (everything in the radar beam) is composed of two classes of objects: objects of interest which are referred to as the target and objects which are not of interest, which are referred to as clutter. Therefore, the scene reflectivity $V$, can be expressed as a superposition of the target reflectivity $T$, and clutter reflectivity $C$ :

$$
V=T+C .
$$

Additionally, in practical radar systems, additive thermal noise is inevitable. Thus, the SAR forward model including clutter and noise can be expressed as:

$$
d(s, t)=\mathcal{F}[T+C](s, t)+n(s, t),
$$

where $n(t, s)$ is the additive thermal noise due to the receiver.

Without loss of generality we assume that both the noise and clutter are zero-mean processes, $E[C(\boldsymbol{x})]=$ $E[n(s, t)]=0$, where $E$ represents the expectation operator. The mean of the target, $E[T(\boldsymbol{x})]$, which is represented as $\mu(\boldsymbol{x})$, is not necessarily spatially constant, however, we make the assumption that $T(\boldsymbol{x})-\mu(\boldsymbol{x})$ and $C(\boldsymbol{x})$ are wide-sense stationary processes. The second order statistics of the target and clutter are defined as follows:

$$
\begin{aligned}
& E\left[\left(T(\boldsymbol{x})-\mu\left(\boldsymbol{x}^{\prime}\right)\right) \overline{\left(T\left(\boldsymbol{x}^{\prime}\right)-\mu\left(\boldsymbol{x}^{\prime}\right)\right)}\right]=\mathcal{C}_{T}\left(\boldsymbol{x}, \boldsymbol{x}^{\prime}\right), \\
& E\left[\left(C(\boldsymbol{x}) \overline{C\left(\boldsymbol{x}^{\prime}\right)}\right]=\mathcal{R}_{C}\left(\boldsymbol{x}, \boldsymbol{x}^{\prime}\right) .\right.
\end{aligned}
$$

We can also represent these statistics from (5) and (6) in terms of their Fourier Transforms,

$$
\begin{aligned}
\mathcal{C}_{T}\left(\boldsymbol{x}, \boldsymbol{x}^{\prime}\right) & =\int \mathrm{e}^{-\mathrm{i} 2 \pi\left(\boldsymbol{x} \cdot \boldsymbol{\varsigma}-\boldsymbol{x}^{\prime} \cdot \boldsymbol{\varsigma}^{\prime}\right)} \tilde{S_{T}}\left(\boldsymbol{\varsigma}, \boldsymbol{\varsigma}^{\prime}\right) d \boldsymbol{\varsigma} d \boldsymbol{\varsigma}^{\prime} \\
\mathcal{R}_{C}\left(\boldsymbol{x}, \boldsymbol{x}^{\prime}\right) & =\int \mathrm{e}^{-\mathrm{i} 2 \pi\left(\boldsymbol{x} \cdot \boldsymbol{\varsigma}-\boldsymbol{x}^{\prime} \cdot \boldsymbol{\varsigma}^{\prime}\right)} \tilde{S_{C}}\left(\boldsymbol{\varsigma}, \boldsymbol{\varsigma}^{\prime}\right) d \boldsymbol{\varsigma} d \boldsymbol{\varsigma}^{\prime}
\end{aligned}
$$

We assume that the noise function $n(s, t)$ is statistically uncorrelated in the slow-time parameter $s$ and stationary in fast-time component $t$. Thus its second order statistics can be represented as follows:

$$
E\left[n(s, t) \overline{n\left(s^{\prime}, t^{\prime}\right)}\right]=\mathcal{R}_{N}(s, \tau) \delta\left(s-s^{\prime}\right),
$$

where $\tau=\left|t-t^{\prime}\right|$. The noise statistic can also be represented as follows:

$$
\mathcal{R}_{N}(s, \tau)=\int \mathrm{e}^{-\mathrm{i} 2 \pi v \tau} S_{N}(s, v) d v .
$$

Lastly, we make the assumption that the target, clutter and noise are all mutually statistically independent.

\section{IMAGE FORMATION}

We first discuss the image formation process for bistatic SAR when there is no noise or clutter. We next discuss the case with noise and clutter. 


\subsection{Ideal image formation}

To form an image $\tilde{T}$ of the target scene we use the following filtered-backprojection operator $\mathcal{K}$ :

$$
\tilde{T}=\mathcal{K}[d](\boldsymbol{z}):=\int \mathrm{e}^{\mathrm{i} 2 \pi v\left(t-R_{T R}(s, \mathbf{z}) / c_{0}\right)} Q_{T R}(\boldsymbol{z}, s, v) d(t, s) d t d s .
$$

We assume the filter $Q_{T R}$ satisfies an estimate similar to the one for $A_{T R}$ in (2).

The combination of the forward map $\mathcal{F}$ and the imaging operator $\mathcal{K}$ can be represented as follows:

$$
\mathcal{K} \mathcal{F}[T](\boldsymbol{z})=\int \mathrm{e}^{\mathrm{i} 2 \pi k\left(R_{T R}(s, \mathbf{x})-R_{T R}(s, \mathbf{z})\right)} Q_{T R}(\boldsymbol{z}, s, v) A_{T R}(\boldsymbol{x}, s, v) T(\boldsymbol{x}) d v d s d \boldsymbol{x},
$$

where $\boldsymbol{z}=\left[z_{1}, z_{2}\right], \mathbf{z}=\left[z_{1}, z_{2}, \psi\left(z_{1}, z_{2}\right)\right]^{T}$, and $k=v / c_{0}$.

For a fixed $\boldsymbol{x}$ and $\boldsymbol{z}$ we can rewrite the phase term in (12) as follows:

$$
R_{T R}(s, \boldsymbol{x})-R_{T R}(s, \boldsymbol{z})=(\boldsymbol{x}-\boldsymbol{z}) \cdot \boldsymbol{\Xi}(s, \boldsymbol{x}, \boldsymbol{z})
$$

where,

$$
\boldsymbol{\Xi}(s, \boldsymbol{x}, \boldsymbol{z})=\int_{0}^{1} \nabla R_{T R}(s, \boldsymbol{z}+\lambda(\boldsymbol{x}-\boldsymbol{z})) d \lambda .
$$

We now employ the Stolt change of variables

$$
(v, s) \rightarrow \boldsymbol{\xi}=\frac{v}{c_{0}} \boldsymbol{\Xi}(s, \mathbf{x}, \mathbf{z})
$$

Thus, (12) becomes

$$
\mathcal{K} \mathcal{F}[T](\boldsymbol{z})=\int \mathrm{e}^{\mathrm{i} 2 \pi(\boldsymbol{x}-\boldsymbol{z}) \cdot \boldsymbol{\xi}} Q_{T R}(\boldsymbol{z}, \boldsymbol{\xi}) A_{T R}(\boldsymbol{x}, \boldsymbol{\xi}) \eta(\boldsymbol{x}, \boldsymbol{z}, \boldsymbol{\xi}) T(\boldsymbol{x}) d \boldsymbol{x} d \boldsymbol{\xi}
$$

where $\eta(\boldsymbol{x}, \boldsymbol{z}, \boldsymbol{\xi})=|\partial(s, v) / \partial \boldsymbol{\xi}|$, the Jacobian for the change of variables in (15).

By using the method of stationary phase we can find the main contributors to the integral, or critical points in (16). ${ }^{28}$ Stationary phase calculations show that the critical points $\mathbf{z}$, are those that have the same bistatic range and same bistatic Doppler as $\mathbf{x} .{ }^{14}$ If we make the further assumption that the antenna beams are focused to one side of their trajectories we can assume that the only critical points are when $\boldsymbol{z}=\boldsymbol{x} .{ }^{14}$ Thus,

$$
\boldsymbol{\Xi}(s, \boldsymbol{z}, \boldsymbol{z})=\left[J\left(z_{1}, z_{2}\right)\right]^{T}\left[\left(\widehat{\gamma_{T}-\mathbf{z}}\right)+\left(\widehat{\gamma_{R}-\mathbf{z}}\right)\right]
$$

where

$$
\begin{aligned}
& J\left(z_{1}, z_{2}\right)=\left[\begin{array}{ll}
\partial \mathbf{z} / \partial z_{1} & \partial \mathbf{z} / \partial z_{2}
\end{array}\right] \\
& =\left[\begin{array}{cc}
1 & 0 \\
0 & 1 \\
\partial \psi / \partial z_{1} & \partial \psi / \partial z_{2}
\end{array}\right] .
\end{aligned}
$$

In order to properly reconstruct the target $T$, the filter $Q_{T R}$ for this case must be the following:

$$
Q_{T R}(\boldsymbol{z}, \boldsymbol{\xi})=\chi_{\Omega_{\boldsymbol{z}}}(\boldsymbol{\xi}) \frac{\overline{A_{T R}(\boldsymbol{z}, \boldsymbol{\xi})}}{\left|A_{T R}(\boldsymbol{z}, \boldsymbol{\xi})\right|^{2}} \frac{1}{\eta(\boldsymbol{z}, \boldsymbol{z}, \boldsymbol{\xi})},
$$


where $\chi_{\Omega_{\boldsymbol{z}}}(\boldsymbol{\xi})$ is the characteristic function of $\Omega_{\boldsymbol{z}}$, the set of Fourier components that the target $T$ can be reconstructed from, and zero outside. $\Omega_{z}$ is referred to as the data collection manifold and is determined by the flight path and frequency of the transmitted waveform. ${ }^{24}$ It is defined as the following set: ${ }^{14}$

$$
\Omega_{\boldsymbol{z}}=\left\{\boldsymbol{\xi}=\frac{v}{c_{0}} \boldsymbol{\Xi}(s, \boldsymbol{z}, \boldsymbol{z}) \mid A_{T R}(\boldsymbol{z}, s, v) \neq 0\right\} .
$$

\subsection{Derivation of the Optimal Filter $Q_{T R}$}

For the best possible image of the scene (in the mean square sense) we would like to define a filter $Q_{T R}$, so that it would yield an image that is close to the following process:

$$
T_{\Omega}(\boldsymbol{z})=I_{\Omega} T=\int_{\Omega_{\boldsymbol{z}}} \mathrm{e}^{\mathrm{i} 2 \pi(\boldsymbol{x}-\boldsymbol{z}) \cdot \boldsymbol{\xi}} \chi_{\Omega_{\boldsymbol{z}}}(\boldsymbol{\xi}) T(\boldsymbol{x}) d \boldsymbol{x} d \boldsymbol{\xi} .
$$

We will design the filter $Q_{T R}$, so the MSE is reduced. The MSE is defined as follows:

$$
\mathcal{J}\left(Q_{T R}\right)=E\left[\int\left|\mathcal{K}[\mathcal{F}[V]+n](\boldsymbol{z})-I_{\Omega}[T](\boldsymbol{z})\right|^{2} d \boldsymbol{z}\right] .
$$

To simplify the notation we write the expectation as an inner-product $\langle\cdot, \cdot\rangle$ :

$$
\begin{aligned}
\mathcal{J}\left(Q_{T R}\right) & =\left\langle\left(\mathcal{K F}-I_{\Omega}\right)[T]+\mathcal{K} \mathcal{F}[C]+\mathcal{K} n,\left(\mathcal{K F}-I_{\Omega}\right)[T]+\mathcal{K} \mathcal{F}[C]+\mathcal{K}[n]\right\rangle \\
& =\mathcal{J}_{T}\left(Q_{T R}\right)+\mathcal{J}_{C}\left(Q_{T R}\right)+\mathcal{J}_{N}\left(Q_{T R}\right)
\end{aligned}
$$

where

$$
\begin{aligned}
\mathcal{J}_{T}\left(Q_{T R}\right) & =\left\langle\left(\mathcal{K} \mathcal{F}-I_{\Omega}\right)[T],\left(\mathcal{K F}-I_{\Omega}\right)[T]\right\rangle \\
\mathcal{J}_{C}\left(Q_{T R}\right) & =\langle\mathcal{K} \mathcal{F}[C], \mathcal{K} \mathcal{F}[C]\rangle \\
\mathcal{J}_{N}\left(Q_{T R}\right) & =\langle\mathcal{K}[n], \mathcal{K}[n]\rangle
\end{aligned}
$$

The cross terms in (23) disappear due to the assumption that the target, clutter and noise are mutually uncorrelated.

Note that for an integral operator $\mathcal{G}$ with $\operatorname{kernel} G$

$$
\begin{aligned}
\langle\mathcal{G}[f], \mathcal{G}[f]\rangle & =\left\langle\int G(\boldsymbol{z}, \boldsymbol{x}) f(\boldsymbol{x}) d \boldsymbol{x}, \overline{\left.\int G\left(\boldsymbol{z}, \boldsymbol{x}^{\prime}\right) f\left(\boldsymbol{x}^{\prime}\right) d \boldsymbol{x}^{\prime}\right\rangle}\right. \\
& =\int G(\boldsymbol{z}, \boldsymbol{x}) \overline{G\left(\boldsymbol{z}, \boldsymbol{x}^{\prime}\right)} \mathcal{R}_{f}\left(\boldsymbol{x}, \boldsymbol{x}^{\prime}\right) d \boldsymbol{x} d \boldsymbol{x}^{\prime} d \boldsymbol{z},
\end{aligned}
$$

where the bar denotes a complex conjugate, $f$ is a general random function and $\mathcal{R}_{f}$ is its autocorrelation function. Using (27), we express $\mathcal{J}_{T}\left(Q_{T R}\right)$ and $\mathcal{J}_{C}\left(Q_{T R}\right)$ as follows:

$$
\begin{gathered}
\mathcal{J}_{T}\left(Q_{T R}\right)=\int \mathrm{e}^{\mathrm{i} 2 \pi(\boldsymbol{x}-\boldsymbol{z}) \cdot \boldsymbol{\xi}}\left[Q_{T R}(\boldsymbol{z}, \boldsymbol{\xi}) A_{T R}(\boldsymbol{x}, \boldsymbol{\xi}) \eta(\boldsymbol{x}, \boldsymbol{z}, \boldsymbol{\xi})-\chi_{\Omega}\right]\left(\mathcal{C}_{T}\left(\boldsymbol{x}, \boldsymbol{x}^{\prime}\right)+\mu(\boldsymbol{x}) \overline{\mu\left(\boldsymbol{x}^{\prime}\right)}\right) d \boldsymbol{\xi} d \boldsymbol{x} \\
\times \int \mathrm{e}^{-\mathrm{i} 2 \pi\left(\boldsymbol{x}^{\prime}-\boldsymbol{z}\right) \cdot \boldsymbol{\xi}^{\prime}}\left[\overline{Q_{T R}\left(\boldsymbol{z}, \boldsymbol{\xi}^{\prime}\right) A_{T R}\left(\boldsymbol{x}^{\prime}, \boldsymbol{\xi}^{\prime}\right) \eta\left(\boldsymbol{x}^{\prime}, \boldsymbol{z}, \boldsymbol{\xi}^{\prime}\right)-\chi_{\Omega}}\right] d \boldsymbol{\xi}^{\prime} d \boldsymbol{x}^{\prime} d \boldsymbol{z} \\
\mathcal{J}_{C}\left(Q_{T R}\right)=\int \mathrm{e}^{\mathrm{i} 2 \pi(\boldsymbol{x}-\boldsymbol{z}) \cdot \boldsymbol{\xi}}\left[Q_{T R}(\boldsymbol{z}, \boldsymbol{\xi}) A_{T R}(\boldsymbol{x}, \boldsymbol{\xi}) \eta(\boldsymbol{x}, \boldsymbol{z}, \boldsymbol{\xi})\right] \mathcal{R}_{C}\left(\boldsymbol{x}, \boldsymbol{x}^{\prime}\right) d \boldsymbol{\xi} d \boldsymbol{x} \\
\times \int \mathrm{e}^{-\mathrm{i} 2 \pi\left(\boldsymbol{x}^{\prime}-\boldsymbol{z}\right) \cdot \boldsymbol{\xi}^{\prime}}\left[\overline{Q_{T R}\left(\boldsymbol{z}, \boldsymbol{\xi}^{\prime}\right) A_{T R}\left(\boldsymbol{x}^{\prime}, \boldsymbol{\xi}^{\prime}\right) \eta\left(\boldsymbol{x}^{\prime}, \boldsymbol{z}, \boldsymbol{\xi}^{\prime}\right)}\right] d \boldsymbol{\xi}^{\prime} d \boldsymbol{x}^{\prime} d \boldsymbol{z} .
\end{gathered}
$$


We use (9) and (10) to express $\mathcal{J}_{N}(Q)$ as follows:

$$
\begin{aligned}
\mathcal{J}_{N}\left(Q_{T R}\right) & =\int \mathrm{e}^{\mathrm{i} 2 \pi v\left(t-R_{T R}(s, \mathbf{z}) / c_{0}\right)} Q_{T R}(\boldsymbol{z}, s, v) \mathcal{R}_{n}\left(t, s, t^{\prime}, s^{\prime}\right) \\
& =\int\left|Q_{T R}(\boldsymbol{z}, s, v)\right|^{2} S_{N}(w, s) d s d w d \boldsymbol{z} .
\end{aligned}
$$

Note that the MSE can also be further broken up into variance and bias terms,

$$
\mathcal{J}\left(Q_{T R}\right)=\mathcal{V}\left(Q_{T R}\right)+\mathcal{B}\left(Q_{T R}\right),
$$

where $\mathcal{V}\left(Q_{T R}\right)$ is the variance and $\mathcal{B}\left(Q_{T R}\right)$ is the bias. The bias term comes from $\mathcal{J}_{T}\left(Q_{T R}\right)$, which can be expressed as

$$
\mathcal{J}_{T}\left(Q_{T R}\right)=\tilde{\mathcal{J}}_{T}\left(Q_{T R}\right)+\mathcal{B}\left(Q_{T R}\right)
$$

where $\tilde{\mathcal{J}}_{T}\left(Q_{T R}\right)$ and $\mathcal{B}\left(Q_{T R}\right)$ can be expressed as the following:

$$
\begin{aligned}
\tilde{\mathcal{J}}_{T}\left(Q_{T R}\right)=\int \mathrm{e}^{\mathrm{i} 2 \pi(\boldsymbol{x}-\boldsymbol{z}) \cdot \boldsymbol{\xi}}\left[Q_{T R}(\boldsymbol{z}, \boldsymbol{\xi})\right. & \left.A_{T R}(\boldsymbol{x}, \boldsymbol{\xi}) \eta(\boldsymbol{x}, \boldsymbol{z}, \boldsymbol{\xi})-\chi_{\Omega}\right] \mathcal{C}_{T}\left(\boldsymbol{x}, \boldsymbol{x}^{\prime}\right) d \boldsymbol{\xi} d \boldsymbol{x} \\
& \left.\times \int \mathrm{e}^{-\mathrm{i} 2 \pi\left(\boldsymbol{x}^{\prime}-\boldsymbol{z}\right) \cdot \boldsymbol{\xi}^{\prime}} \overline{\left[Q_{T R}\left(\boldsymbol{z}, \boldsymbol{\xi}^{\prime}\right) A_{T R}\left(\boldsymbol{x}^{\prime}, \boldsymbol{\xi}^{\prime}\right) \eta\left(\boldsymbol{x}^{\prime}, \boldsymbol{z}, \boldsymbol{\xi}^{\prime}\right)-\chi_{\Omega}\right.}\right] d \boldsymbol{\xi}^{\prime} d \boldsymbol{x}^{\prime} d \boldsymbol{z},
\end{aligned}
$$

and

$$
\begin{aligned}
\mathcal{B}\left(Q_{T R}\right)=(2 \pi)^{2} \int \mathrm{e}^{\mathrm{i} 2 \pi(\boldsymbol{x}-\boldsymbol{z}) \cdot \boldsymbol{\xi}}\left[Q_{T R}(\boldsymbol{z}, \boldsymbol{\xi}) A_{T R}(\boldsymbol{x}, \boldsymbol{\xi}) \eta(\boldsymbol{x}, \boldsymbol{z}, \boldsymbol{\xi})-\chi_{\Omega}\right] \mu(\boldsymbol{x}) \overline{\mu\left(\boldsymbol{x}^{\prime}\right)} d \boldsymbol{\xi} d \boldsymbol{x} \\
\left.\quad \times \int \mathrm{e}^{-\mathrm{i} 2 \pi\left(\boldsymbol{x}^{\prime}-\boldsymbol{z}\right) \cdot \boldsymbol{\xi}^{\prime}} \overline{\left[Q_{T R}\left(\boldsymbol{z}, \boldsymbol{\xi}^{\prime}\right) A_{T R}\left(\boldsymbol{x}^{\prime}, \boldsymbol{\xi}^{\prime}\right) \eta\left(\boldsymbol{x}^{\prime}, \boldsymbol{z}, \boldsymbol{\xi}^{\prime}\right)-\chi_{\Omega}\right.}\right] d \boldsymbol{\xi}^{\prime} d \boldsymbol{x}^{\prime} d \boldsymbol{z} .
\end{aligned}
$$

In order to find a close form expression for the filter $Q_{T R}$, there must be a trade-off between variance and bias. In essence a minimum variance estimate will be biased for this problem. ${ }^{25}$ For this work we are concerned with reducing the MSE through finding the minimum variance estimate. Thus we drop the bias term and do not use it in our further calculations.

We use the method of stationary phase to identify the main contributions to $\mathcal{V}\left(Q_{T R}\right)$. First we substitute (7) and (8) into (33) and (29) respectivly. This results in the following phase term for $\tilde{\mathcal{J}}_{T}\left(Q_{T R}\right)$ and $\mathcal{J}_{C}\left(Q_{T R}\right)$ :

$$
\phi=\boldsymbol{x} \cdot(\boldsymbol{\xi}-\boldsymbol{\varsigma})+\boldsymbol{z} \cdot\left(\boldsymbol{\xi}^{\prime}-\boldsymbol{\xi}\right)+\boldsymbol{x}^{\prime} \cdot\left(\boldsymbol{\varsigma}^{\prime}-\boldsymbol{\xi}^{\prime}\right) .
$$

By carrying out the stationary phase analysis we can find the resultant critical points,

$$
\begin{aligned}
& 0=\nabla_{\boldsymbol{x}^{\prime}} \phi=\boldsymbol{\varsigma}^{\prime}-\boldsymbol{\xi}^{\prime} \rightarrow \boldsymbol{\varsigma}^{\prime}=\boldsymbol{\xi}^{\prime} \\
& 0=\nabla_{\boldsymbol{\xi}^{\prime}} \phi=\boldsymbol{z}-\boldsymbol{x}^{\prime} \rightarrow \boldsymbol{z}=\boldsymbol{x}^{\prime} \\
& 0=\nabla_{\boldsymbol{x}} \phi=\boldsymbol{\xi}-\boldsymbol{\varsigma} \rightarrow \boldsymbol{\xi}=\boldsymbol{\varsigma} \\
& 0=\nabla_{\boldsymbol{\xi}} \phi=\boldsymbol{x}-\boldsymbol{z} \rightarrow \boldsymbol{x}-\boldsymbol{z} .
\end{aligned}
$$

With our previous substitutions and stationary phase analysis we can now write the variance terms $\tilde{\mathcal{J}}_{T}\left(Q_{T R}\right)$ and $\mathcal{J}_{C}\left(Q_{T R}\right)$ as the following: 


$$
\begin{gathered}
\tilde{\mathcal{J}}_{T}\left(Q_{T R}\right) \approx \int \mathrm{e}^{\mathrm{i} 2 \pi \boldsymbol{z} \cdot\left(\boldsymbol{\xi}^{\prime}-\boldsymbol{\xi}\right)}\left[Q_{T R}(\boldsymbol{z}, \boldsymbol{\xi}) A_{T R}(\boldsymbol{z}, \boldsymbol{\xi}) \eta(\boldsymbol{z}, \boldsymbol{z}, \boldsymbol{\xi})-\chi_{\Omega_{\boldsymbol{z}}}\right] \tilde{S}_{T}\left(\boldsymbol{\xi}, \boldsymbol{\xi}^{\prime}\right) \\
\times\left[\overline{Q_{T R}\left(\boldsymbol{z}, \boldsymbol{\xi}^{\prime}\right) A_{T R}\left(\boldsymbol{z}, \boldsymbol{\xi}^{\prime}\right) \eta\left(\boldsymbol{z}, \boldsymbol{z}, \boldsymbol{\xi}^{\prime}\right)-\chi_{\Omega_{\boldsymbol{z}}}}\right] d \xi d \boldsymbol{\xi}^{\prime} d \boldsymbol{z} \\
\mathcal{J}_{C}\left(Q_{T R}\right) \approx \int \mathrm{e}^{\mathrm{i} 2 \pi \boldsymbol{z} \cdot\left(\boldsymbol{\xi}^{\prime}-\boldsymbol{\xi}\right)}\left[Q_{T R}(\boldsymbol{z}, \boldsymbol{\xi}) A_{T R}(\boldsymbol{z}, \boldsymbol{\xi}) \eta(\boldsymbol{z}, \boldsymbol{z}, \boldsymbol{\xi})\right] \tilde{S}_{C}\left(\boldsymbol{\xi}, \boldsymbol{\xi}^{\prime}\right) \\
\times\left[\overline{Q_{T R}\left(\boldsymbol{z}, \boldsymbol{\xi}^{\prime}\right) A_{T R}\left(\boldsymbol{z}, \boldsymbol{\xi}^{\prime}\right) \eta\left(\boldsymbol{z}, \boldsymbol{z}, \boldsymbol{\xi}^{\prime}\right)}\right] d \boldsymbol{\xi} d \boldsymbol{\xi}^{\prime} d \boldsymbol{z} .
\end{gathered}
$$

If we make the assumption that the second order statistics are stationary, the power spectral densities for the target and clutter, $S_{T}(\boldsymbol{\xi})$ and $S_{C}(\boldsymbol{\xi})$, are defined as follows:

$$
\begin{aligned}
& \tilde{S}_{T}\left(\boldsymbol{\xi}, \boldsymbol{\xi}^{\prime}\right)=S_{T}(\boldsymbol{\xi}) \delta\left(\boldsymbol{\xi}-\boldsymbol{\xi}^{\prime}\right) \\
& \tilde{S}_{C}\left(\boldsymbol{\xi}, \boldsymbol{\xi}^{\prime}\right)=S_{C}(\boldsymbol{\xi}) \delta\left(\boldsymbol{\xi}-\boldsymbol{\xi}^{\prime}\right) .
\end{aligned}
$$

With the stationarity assumption, the expressions for $\tilde{\mathcal{J}}_{T}\left(Q_{T R}\right)$ and $\mathcal{J}_{C}\left(Q_{T R}\right)$ become

$$
\begin{aligned}
\tilde{\mathcal{J}}_{T}\left(Q_{T R}\right) & =\int\left|Q_{T R}(\boldsymbol{z}, \boldsymbol{\xi}) A_{T R}(\boldsymbol{z}, \boldsymbol{\xi}) \eta(\boldsymbol{z}, \boldsymbol{z}, \boldsymbol{\xi})-\chi_{\Omega_{\boldsymbol{z}}}\right|^{2} S_{T}(\boldsymbol{\xi}) d \boldsymbol{\xi} d \boldsymbol{z} \\
\mathcal{J}_{C}\left(Q_{T R}\right) & =\int\left|Q_{T R}(\boldsymbol{z}, \boldsymbol{\xi}) A_{T R}(\boldsymbol{z}, \boldsymbol{\xi}) \eta(\boldsymbol{z}, \boldsymbol{z}, \boldsymbol{\xi})\right|^{2} S_{C}(\boldsymbol{\xi}) d \boldsymbol{\xi} d \boldsymbol{z}
\end{aligned}
$$

If we extend the change of variables stated in (15) and stationary phase analysis to (30), the expression for $\mathcal{J}_{N}\left(Q_{T R}\right)$ becomes

$$
\mathcal{J}_{N}\left(Q_{T R}\right)=\int\left|Q_{T R}(\boldsymbol{z}, \boldsymbol{\xi})\right|^{2} \eta(\boldsymbol{z}, \boldsymbol{z}, \boldsymbol{\xi}) S_{N}(\boldsymbol{\xi}) d \boldsymbol{\xi} d \boldsymbol{z} .
$$

To determine the optimal filter to minimize the variance, we derive the variational derivative of $\mathcal{V}(Q)$ with respect to $Q$ as follows:

$$
\left.\frac{d}{d \epsilon} \mathcal{V}\left(Q_{T R}+\epsilon Q_{\epsilon}\right)\right|_{\epsilon=0}=\left.\frac{d}{d \epsilon} \tilde{\mathcal{J}}_{T}\left(Q_{T R}+\epsilon Q_{\epsilon}\right)\right|_{\epsilon=0}+\left.\frac{d}{d \epsilon} \mathcal{J}_{C}\left(Q_{T R}+\epsilon Q_{\epsilon}\right)\right|_{\epsilon=0}+\left.\frac{d}{d \epsilon} \mathcal{J}_{N}\left(Q_{T R}+\epsilon Q_{\epsilon}\right)\right|_{\epsilon=0}
$$

The variational derivative of $\tilde{\mathcal{J}}_{T}(Q)$ is

$$
\left.\frac{d}{d \epsilon} \tilde{\mathcal{J}}_{T}\left(Q_{T R}+\epsilon Q_{\epsilon}\right)\right|_{\epsilon=0}=\int\left(\overline{Q_{T R} A_{T R} \eta-\chi_{\Omega_{z}}}\right) S_{T} Q_{\epsilon} A_{T R} \eta d \boldsymbol{\varsigma} d \boldsymbol{z}+\int \overline{Q_{\epsilon} A_{T R} \eta} S_{T}\left(Q_{T R} A_{T R} \eta-\chi_{\Omega_{\boldsymbol{z}}}\right) d \boldsymbol{\varsigma} d \boldsymbol{z} .
$$

Because, $S_{T}$ is real, (44) becomes

$$
\left.\frac{d}{d \epsilon} \tilde{\mathcal{J}}_{T}\left(Q_{T R}+\epsilon Q_{\epsilon}\right)\right|_{\epsilon=0}=2 \operatorname{Re} \int \overline{Q_{\epsilon} A_{T R} \eta} S_{T}\left(Q_{T R} A_{T R} \eta-\chi_{\Omega_{\boldsymbol{z}}}\right) d \boldsymbol{\varsigma} d \boldsymbol{z}
$$

We can follow the same procedure for $\mathcal{J}_{C}\left(Q_{T R}\right)$ and $\mathcal{J}_{N}\left(Q_{T R}\right)$ and find the following: 


$$
\begin{aligned}
\left.\frac{d}{d \epsilon} \mathcal{J}_{C}\left(Q_{T R}+\epsilon Q_{\epsilon}\right)\right|_{\epsilon=0} & =2 \operatorname{Re} \int \overline{Q_{\epsilon} A_{T R} \eta} S_{C}\left(Q_{T R} A_{T R} \eta\right) d \boldsymbol{\varsigma} d \boldsymbol{z} \\
\left.\frac{d}{d \epsilon} \mathcal{J}_{N}\left(Q_{T R}+\epsilon Q_{\epsilon}\right)\right|_{\epsilon=0} & =2 \operatorname{Re} \int \overline{Q_{\epsilon}} S_{N} Q_{T R} \eta d \boldsymbol{\varsigma} d \boldsymbol{z}
\end{aligned}
$$

To find the the filter $Q_{T R}$ that yields the minimum variance, we combine the right hand sides of (45), (46) and (47)

$$
\left.\frac{d}{d \epsilon} \mathcal{V}\left(Q_{T R}+\epsilon Q_{\epsilon}\right)\right|_{\epsilon=0}=2 \operatorname{Re} \int\left[\overline{Q_{\epsilon} A_{T R} \eta}\left(Q_{T R} A_{T R} \eta-\chi_{\Omega_{z}}\right) S_{T}+\overline{Q_{\epsilon} A_{T R} \eta}\left(Q_{T R} A_{T R} \eta\right) S_{C}+\overline{Q_{\epsilon}} Q \eta S_{N}\right] d \boldsymbol{\varsigma} d \boldsymbol{z}
$$

If the right hand side of (48) is to be zero for all $Q_{\epsilon}$, then the following equation must hold

$$
\overline{A_{T R} \eta}\left(Q_{T R} A_{T R} \eta-\chi_{\Omega_{z}}\right)+\overline{A_{T R} \eta}\left(Q_{T R} A_{T R} \eta\right) S_{C}+Q_{T R} \eta S_{N}=0
$$

Thus,

$$
Q_{T R}(\boldsymbol{z}, \boldsymbol{\xi})=\frac{\chi_{\Omega_{\boldsymbol{z}}}(\boldsymbol{\xi}) \overline{A_{T R}(\boldsymbol{z}, \boldsymbol{\xi}) \eta(\boldsymbol{z}, \boldsymbol{z}, \boldsymbol{\xi})} S_{T}(\boldsymbol{\xi})}{\left|A_{T R}(\boldsymbol{z}, \boldsymbol{\xi}) \eta(\boldsymbol{z}, \boldsymbol{z}, \boldsymbol{\xi})\right|^{2}\left[S_{T}(\boldsymbol{\xi})+S_{C}(\boldsymbol{\xi})\right]+\eta(\boldsymbol{z}, \boldsymbol{z}, \boldsymbol{\xi}) S_{N}(\boldsymbol{\xi})}, \boldsymbol{\xi} \in \Omega_{\boldsymbol{z}}
$$

A form that is more similar to the deterministic filter seen in (19) is the following:

$$
Q_{T R}(\boldsymbol{z}, \boldsymbol{\xi})=\left[\frac{\overline{A_{T R}(\boldsymbol{z}, \boldsymbol{\xi})}}{\left|A_{T R}(\boldsymbol{z}, \boldsymbol{\xi})\right|^{2} \eta(\boldsymbol{z}, \boldsymbol{z}, \boldsymbol{\xi})} \chi_{\Omega_{\boldsymbol{z}}}(\boldsymbol{\xi})\right]\left[\frac{S_{T}(\boldsymbol{\xi})}{\left[S_{T}(\boldsymbol{\xi})+S_{C}(\boldsymbol{\xi})\right]+\left[\frac{1}{\left|A_{T R}(\boldsymbol{z}, \boldsymbol{\xi})\right|^{2} \overline{\bar{\eta}(\boldsymbol{z}, \boldsymbol{z}, \boldsymbol{\xi})}}\right] S_{N}(\boldsymbol{\xi})}\right], \boldsymbol{\xi} \in \Omega_{\boldsymbol{z}}
$$

\section{NUMERICAL SIMULATIONS}

In this section we present our numerical simulations along with the results to illustrate the theory as well as to demonstrate the performance of the algorithm. We first discuss our simulation set up and then our results.

\subsection{Simulation set up}

For the numerical simulations we made the following assumptions: first, we assumed that the earth's surface is flat, and we chose the amplitude function $A_{T R} \equiv 1$. This specific amplitude function corresponds to isotropic transmitters and receivers using a delta function as the transmit waveform.

In the numerical simulations we have considered a scene of size $[0,22] \times[0,22] \mathrm{km}^{2}$, with two rectangles with centers located at $(8.8,12) \mathrm{km}$ and $(15.4,10) \mathrm{km}$, as seen in Figure 1 . The scene has been discretized to a $64 \times 64$ grid of pixels where $(0,0,0) \mathrm{km}$ and $(22,22,0) \mathrm{km}$ correspond to pixel numbers $(1,1)$ and $(64,64)$ respectively. In the experiments, we use a circular flight trajectory, $\gamma(s)=(11+22 \cos s, 11+22 \sin s, 6.5) \mathrm{km}$, which is uniformly sampled by 512 points in $s \in[0,2 \pi]$. For our specific experiments, for which results are shown in Figure sets 2 and 3 , the receiver trajectory is set to be $\gamma_{T}(s)=\gamma(s)$ and the transmitter trajectory is $\gamma_{T}(s)=\gamma(s+\pi / 4)$.

The objective of our simulations is to study the performance of our reconstruction algorithm in the presence of noise and clutter as compared to the deterministic reconstruction that was presented in. ${ }^{14}$ To do this we needed to determine the spectrum of the target which was determined by numerically correlating the target image with itself and then taking the FFT of the resultant correlation. The noise and clutter processes were generated from Gaussian white noise using a Karhunen-Loeve transform to shape their spectrums. The spectrum of the clutter was shaped using a high frequency model of the target where 


$$
S_{C}=\frac{1}{4} \sum_{ \pm}\left(S_{T}\left(\varsigma_{1} \pm 8, \varsigma_{2} \pm 8\right)+S_{T}\left(\varsigma_{1} \mp 8, \varsigma_{2} \mp 8\right)\right) .
$$

For the noise process we used the following wideband noise model:

$$
S_{N}(s, v)=\frac{1}{1+|v|^{5}} \frac{\pi / 5}{\sin (\pi / 5)} .
$$

To quantify our results we use the definitions of SCR, SNR and MSE found in. ${ }^{25}$ Definitions of SCR and SNR are as follows:

$$
\begin{gathered}
\mathrm{SCR}=20 \log \frac{\frac{1}{N_{T}} \sum_{i=1}^{N_{T}}\left(T\left(\boldsymbol{x}_{i}\right)-\mu_{T}\right)^{2}}{E[\|C\|]} \mathrm{dB} \\
\mathrm{SNR}=20 \log \frac{\frac{1}{N_{d}} \sum_{i=1}^{N_{d}}\left(d\left(s_{i}, t_{i}\right)-\mu_{d}\right)^{2}}{E[\|n\|]} \mathrm{dB},
\end{gathered}
$$

where $N_{T}$ and $N_{d}$ are the number of grid points and $\mu_{T}$ and $\mu_{d}$ are the mean values of the target scene and its radar data respectively. The MSE is defined as follows:

$$
\mathrm{MSE}=\frac{\sum_{j=1}^{M} \sum_{i=1}^{N_{T}}\left|T\left(\boldsymbol{x}_{i}\right)-\hat{T}_{j}\left(\boldsymbol{x}_{i}\right)\right|^{2}}{N_{T} M}
$$

where $M$ is the number of reconstructions and $\hat{T}_{j}\left(\boldsymbol{x}_{i}\right)$ is the reconstruction for the $j^{\text {th }}$ reconstruction of the noise and clutter processes.

\subsection{Simulation results}

In Figure set 2 the algorithm's performance in the case of cluttered scene where the $\mathrm{SCR}=-30 \mathrm{~dB}$. The performance in the case of noisy data can be seen in Figure set 3 where the SNR $=-30 \mathrm{~dB}$. In both cases we can see a large increase in the quality of the image and are able to make out features of the target that were masked before.

Figure 4(a) shows the MSE and variance of the reconstructed images averaged over ten realizations of clutter using our clutter suppression filter derived and the deterministic filter shown in (19). The same is shown in Figure 4(b) in the case of a noisy received signal with ten different realizations of noise. It is worthy of noting that at high SNR and SCR values the two different procedures yield similar results. The reason for this can be seen in the limiting case for (51) where it is equal to (19), if $S_{C}$ and $S_{N}$ both are zero.

\section{CONCLUSIONS}

In this paper we present a filtered-backprojection scheme for BISAR when the received data are corrupted by noise and clutter. The reconstruction procedure is derived using microlocal techniques in a statistical setting. We show that through the assumption of stationarity of the second order statistics, an explicit filter minimizing the variance between the true and reconstructed scene can be derived.

We performed numerical simulations to show the effectiveness of our method in the presence of noise and clutter. These simulations show a reduction in the variance and MSE of the images as well as enhanced image quality. 


\section{REFERENCES}

[1] Willis, N. J., [Bistatic Radar], Artech House, Norwood, MA (1991).

[2] Horne, A. M. and Yates, G., "Bistatic synthetic aperture radar," Proceedings of the IEEE Radar Confrence , 6-10 (May 2005).

[3] Richards, M. A., [Fundamentals of Radar Signal Processing], McGraw-Hill, New York, NY (2005).

[4] Soumekh, M., [Synthetic Aperture Radar Signal Processing with MATLAB Algorithms], John Wiley and Sons, New York, NY (1999).

[5] Cutrona, L. J., [Radar Handbok], ch. Synthetic Aperture Radar, 207-219, McGraw-Hill, New York, NY, 2nd ed. (1990).

[6] Munson, D.C., J., O'Brien, J., and Jenkins, W., "A tomographic formulation of spotlight-mode synthetic aperture radar," Proceedings of the IEEE 71, 917-925 (Aug. 1983).

[7] Arikan, O. and Munson, D. C., "A tomographic formulation of bistatic synthetic aperture radar," Inter. Conf. on Advances in Communication and Control Systems , 289302 (1988).

[8] Rigling, B., Signal Processing Stratagies for Bistatic Synthetic Aperture Radar, PhD in Electrical engineering, The Ohio State University, Columbus, OH (2003).

[9] Ulander, L. and Frolind, P.-O., "Ultra-wideband sar interferometry," IEEE Transactions on Geoscience and Remote Sensing 36, 1540-1550 (Sep 1998).

[10] Ulander, L. and Hellsten, H., "Low-frequency ultra-wideband array-antenna sar for stationary and moving target imaging," in [Proceedings of Radar Sensor Technology IV], 3704, 149-158, SPIE, Orlando, FL (April 1999).

[11] Nolan, C. J. and Cheney, M., "Synthetic aperture inversion," Inverse Problems 18(1), 221-235 (2002).

[12] Nolan, C. and Cheney, M., "Synthetic aperture inversion for arbitrary flight paths and nonflat topography," IEEE Transactions on Image Processing 12, 1035-1043 (Sept. 2003).

[13] Cheney, M., "Synthetic-aperture assessment of a dispersive surface," International Journal of Imaging Systems and Technology 22, 28-34 (2004).

[14] Yarman, C., Yazici, B., and Cheney, M., "Bistatic synthetic aperture radar imaging for arbitrary flight trajectories," IEEE Transactions on Image Processing 17, 84-93 (Jan. 2008).

[15] Duistermaat, J., [Fourier Integral Operators], Birkhauser, Boston, MA (1996).

[16] Grigis, A. and Sjöstrand, J., [Microlocal Analysis for Differential Operators: An Introduction], Cambridge University Press, Cambridge (1996).

[17] Treves, F., [Introduction to Pseudodifferential and Fourier Integral Operators], vol. 1 and 2, Plenum, New York (1980).

[18] Bleistein, N., Cohen, J. K., and Stockwell, J., [the Mathematics of Multidimensional Seismic Inversion], Springer, New York (2000).

[19] Louis, A. Quinto, E., [Local tomographic methods in SONAR], Springer, New York (2000).

[20] Quinto, E. T., "Singularities of the x-ray transform and limited data tomography in r2 and r3," SIAM J. Math. Anal. 24(5), 1215-1225 (1993).

[21] Beylkin, G., "Imaging of discontinuities in the inverse scattering problem by inversion of a causal generalized radon transform," Journal of Mathematical Physics 26, 99-108 (1985).

[22] Beylkin, G. and Burridge, R., "Linearized inverse scatter problems in acoustics and elasticity," Wave Motion 12, 15-52 (1990).

[23] Nolan, C. J. and W., S. W., "Global solution of a linearized inverse problem for the acoustic wave equation," Communications in Partial Differential Equations 22, 919-52 (1997).

[24] Yazici, B. and Cheney, M., "Synthetic aperture inversion for arbitrary flight paths in the presence of noise and clutter," 2005 IEEE International Radar Conference, 806-810 (May 2005).

[25] Yazici, B., Cheney, M., and Yarman, C. E., "Synthetic-aperture inversion in the presence of noise and clutter," Inverse Problems 22(5), 1705-1729 (2006).

[26] Cetin, M. and Karl, W., "Superresolution and edge-preserving reconstruction of complex-valued synthetic aperture radar images," Proceedings of the International Conference on Image Processing 1, 701-704 vol.1 (September 2000). 
[27] Cetin, M., Karl, W., and Willsky, A., "Edge-preserving image reconstruction for coherent imaging applications," Proceedings of the International Conference on Image Processing 2, II-481-II-484 vol.2 (September 2002).

[28] Papoulis, A., [Signal Analysis], McGraw-Hill, New York (1977).

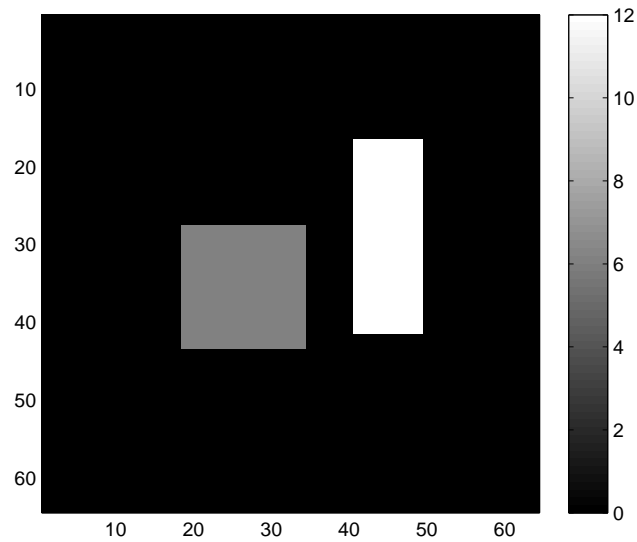

Figure 1. Image of the target without clutter.

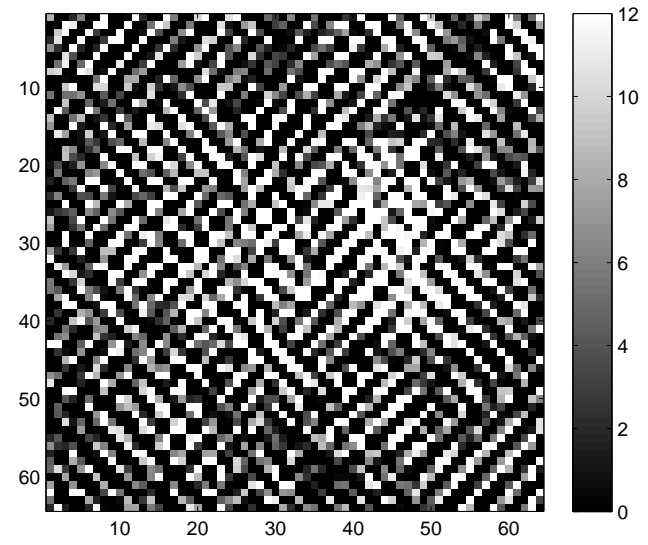

(a)

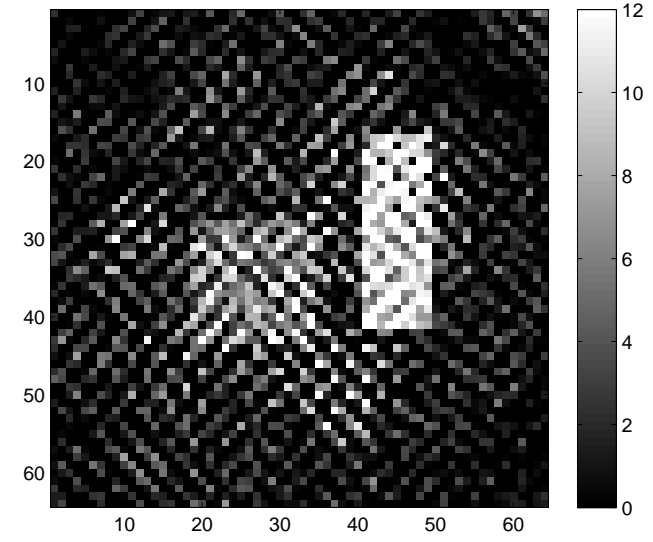

(b)

Figure 2. Left: single reconstruction of a cluttered scene, right: average of ten reconstructions using clutter suppression filter $Q_{T R}$ when $\mathrm{SCR}=-30 \mathrm{~dB}$. 


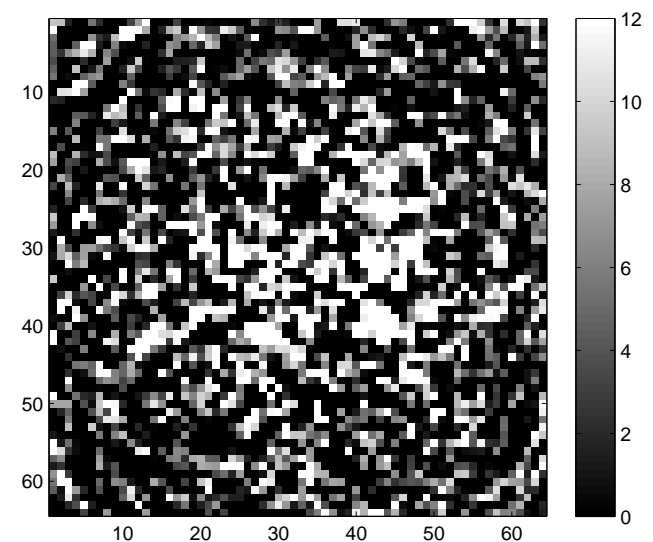

(a)

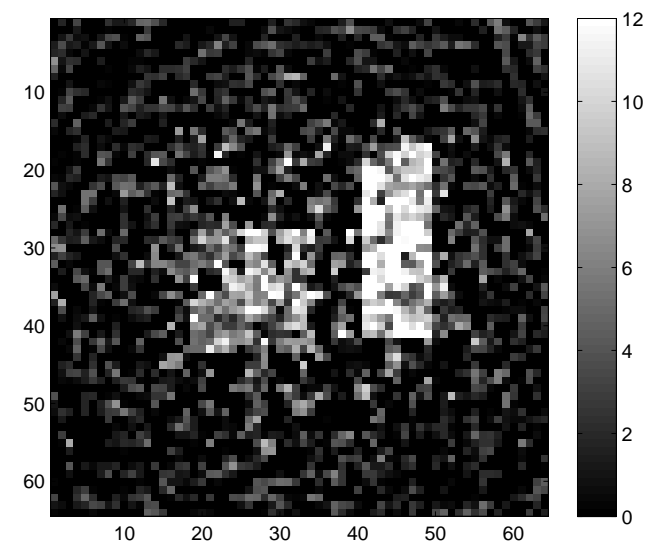

(b)

Figure 3. Left: single reconstruction of target from noisy data, right: average of ten reconstructions using noise suppression filter $Q_{T R}$ when $\mathrm{SNR}=-30 \mathrm{~dB}$.

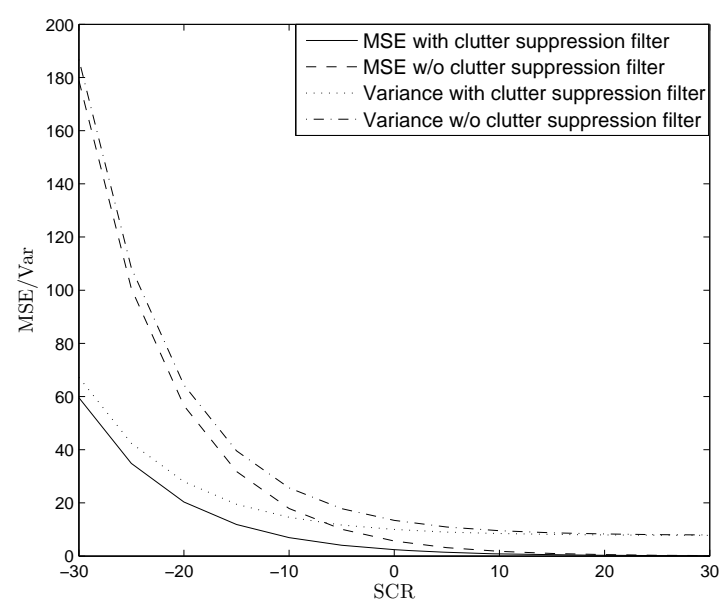

(a)

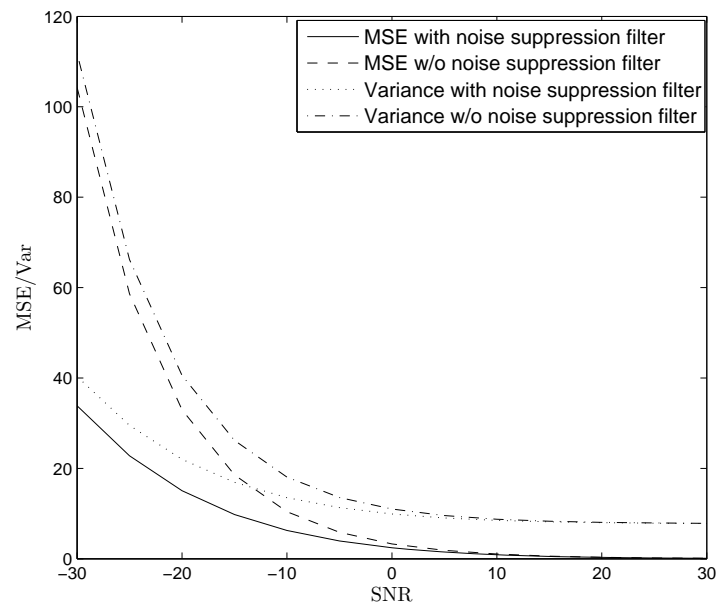

(b)

Figure 4. Left: MSE curve with no noise and variable clutter ratio. Right: MSE curve with no clutter and variable noise. 